\title{
Endoscopic Submucosal Dissection
}

\author{
Filippo Catalano
}

The endoscopic submucosal dissection (ESD) technique was firstly proposed and developed in Japan by Hosokawa for the treatment of mucosal early gastric cancer (EGC). The aim of ESD is to perform en-bloc resection of the lesion to reduce the risk of local recurrence and allow correct histological evaluation to assess curativity criteria.

In this video chapter we demonstrate the main steps in performing an ESD for EGC.

The first step of endoscopic submucosal dissection is represented by marking the normal mucosa around the lesion with at least $5 \mathrm{~mm}$ of free margins using a standard needle knife (Olympus Co., Tokyo, Japan) with a forced $20 \mathrm{~W}$ coagulation current (ICC 200 or VIO 200 ERBE, Tubingen, Germany). The next step is the lifting of the lesion with a submucosal injection; a saline solution mixed with epinephrine $(0.04 \mathrm{mg} / \mathrm{mL})$ and small amount of indigo carmine or methylene blue is used, in order to better recognize the different layers. At this point a circumferential mucosal incision is carried out outside the marking dots with a needle knife in the $60 \mathrm{~W}$ endo-cut mode effect 3 and then completed with an insulation-tipped (IT) knife, in the 60-80 W endo-cut mode effect 3 (ICC 200 ERBE, Tubingen, Germany). The lesion's lateral borders are now free from the rest of the tissue and the submucosal layer underneath the lesion is carefully dissected from the muscle layer with the IT knife. Hemostasis of blood vessels in the submucosal space during the procedure is achieved with the same knife or with hemostatic forceps (Coagrasper, Olympus).

Lastly the specimen is orientated on a plate and is fixed with thin needles circumferentially, on the edge of the resection to allow correct histological evaluation.

\footnotetext{
Supplementary Information The online version of this chapter (https://doi. org/10.1007/978-3-030-73158-8_39) contains supplementary material, which is available to authorized users.
}

\section{F. Catalano $(\square)$}

Digestive Endoscopy Division, Department of Surgery, Borgo Trento Hospital, Verona, Italy e-mail: filippo.catalano@aovr.veneto.it 